Graciela Ortiz

Martha Lucía

Cervantes I

\title{
LA FORMACIÓN CIENTÍFICA EN LOS PRIMEROS AÑOS DE ESCOLARIDAD
}

\author{
Scientific Training during Elementary Education
}

A formação científica nos primeiros anos de escolaridade

RECIBIDO:10DE AGOSTODE 2015

Graciela Ortiz Rivera (Colombia) ${ }^{1}$

Universidad del Atlántico

Máster en Educación

chelin1230@hotmail.com

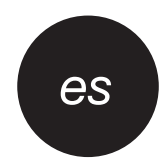

\section{RESUMEN}

El presente artículo plantea una situación existente en muchas aulas de educación inicial donde la ciencia es tratada como un área más contemplada en los planes de estudio y desarrollada mediante temas o contenidos programáticos alejados de la realidad cercana a los estudiantes. Se definen los conceptos de ciencia, curiosidad y algunas habilidades científicas, como clasificación, inferencia, observación, formulación de preguntas y planteamiento de hipótesis, y se hace una reflexión crítica sobre la formación científica durante los primeros años de escolaridad. Además, se expone el tratamiento cultural e institucional que se ha venido dando a la ciencia en las aulas de preescolar y se concluye con un llamado a las maestras y los maestros a que contemplen otras formas de trabajarla con los niños.

Panorama I

PALABRAS CLAVE: ciencia, curiosidad, habilidades científicas
EVALUADO:29DE SEPTIEMBREDE 2015

ACEPTADO:3 DE NOVIEMBRE DE 2015

\section{en}

\section{ABSTRACT}

This article presents a situation that exists in many classrooms where science is regarded as another area to be included in the plans of study in order to be developed through programmatical topics or contents further from the truth students live. We define science and curiosity concepts as well as some scientific abilities such as classification, inference, observation, and formulation of questions and hypotheses. We also criticize the scientific training received during elementary education as well as the cultural and institutional treatment given to science during preschool education. We conclude by suggesting teachers to innovate the way they teach science to children.

KEYWORDS: Science, curiosity, scientific abilities.

\author{
Martha Lucía Cervantes Coronado \\ $(\text { Colombia })^{2}$ \\ Universidad del Atlántico \\ Máster en Educación \\ marthacervantes1302@hotmail.com
}

\section{por}

\section{RESUMO}

O presente artigo apresenta uma situação existente em muitas aulas de educação inicial onde a ciência é tratada como uma área mais contemplada nos planos de estudo e desenvolvida mediante temas ou conteúdos programáticos longe da realidade próxima aos estudantes. Definem-se os conceitos de ciência, curiosidade e algumas habilidades científicas, como classificação, inferência, observação, formulação de preguntas e apresentação de hipóteses, e se faz uma reflexão crítica sobre a formação científica durante os primeiros anos de escolaridade. Além do mais, se expõe o tratamento cultural e institucional que se tem vindo dando à ciência nas aulas de pré-escolar e se conclui com um chamado às mestras e os mestres para que contemplem outras formas de trabalhá-la com as crianças.

PALAVRAS CHAVE: ciência, curiosidade, habilidades científicas. 
Es necesario entonces ofrecer una sólida formación en ciencias que debe comenzar en los primeros años de escolaridad, que acentúe el desarrollo de actitudes y habilidades científicas, sin descuidar los contenidos de tipo teórico, mediante proyectos y experiencias que permitan a los niños expresar su curiosidad natural en la cotidianidad de la escuela. Estas vivencias deben constituirse en experiencias investigativas en las que tanto docentes como estudiantes interactúen en la búsqueda de respuestas a sus propios interrogantes. Tal como lo plantea Tonucci (1995), la investigación existe cuando se está frente a un problema cuya solución es necesaria y el método de la investigación impone que se resuelva el problema utilizando los conocimientos anteriores en forma nueva, creativa.

la Organización de las Naciones Unidas para la Educación, la Ciencia y la Cultura (Unesco) y el Consejo Internacional para la Ciencia: "Para que un país tenga la capacidad de abastecer las necesidades básicas de su población, la educación en ciencia y tecnología es una necesidad estratégica" (Unesco, 1999).

En Colombia, el Ministerio de Educación Nacional (MEN) propone, en los Estándares básicos de competencias en ciencias naturales y sociales:

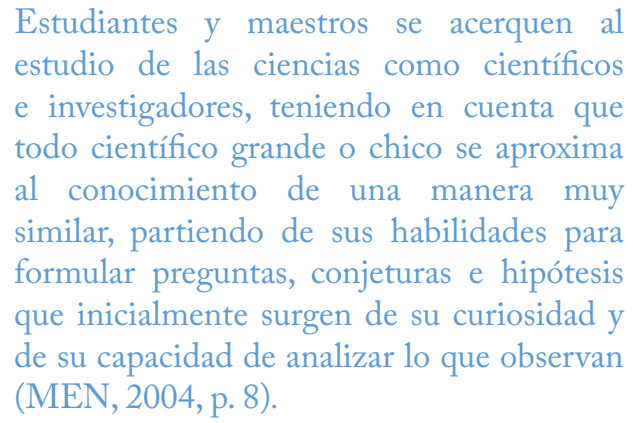

Pese a esto, la realidad demuestra que algo está fallando en la formación científica no solo en los primeros años de escolaridad, sino a lo largo de todo el proceso educativo. En este sentido, Claxton afirma:

Muchos niños y jóvenes se aburren en las clases de ciencia y pocos se interesan y llegan a culminar una carrera científica, al grado que existe un verdadero déficit de científicos en muchos países. Después de varios años de recibir una formación científica, un alto porcentaje de jóvenes carece de los conocimientos y habilidades científicas básicas (1994, p. 9).
En este artículo, se aborda el concepto de ciencia como una actividad humana y no como un cúmulo de conocimientos y se concibe la curiosidad como una actitud que acerca al niño al conocimiento científico. Igualmente se muestra una percepción global de la manera como se presenta la ciencia en la escuela en especial en la etapa inicial de la educación formal. Finalmente, se expone el tema de las habilidades científicas en los niños, definiendo aquellas que se consideran necesarias para iniciar el abordaje de la ciencia.

\section{LA CIENCIA CONSIDERADA COMO ACTIVIDAD HUMANA}

Etimológicamente la palabra ciencia proviene del latín scientia, cuyo significado es conocimiento o saber. Desde esta definición, se designa ciencia a todo el conocimiento adquirido a través del estudio o de la práctica, basado en determinados principios. El Diccionario de la lengua española la define como "conjunto de conocimientos obtenidos mediante la observación y el razonamiento, sistemáticamente estructurados, de los que se deducen principios y leyes generales" (http://lema.rae. es/drae/?val=ciencia). Sin embargo, tal como lo afirma Coleto (2009), en los últimos años se ha pasado de considerar que la ciencia es un conjunto organizado y validado de conocimientos que explican cómo es el mundo en que vivimos a creer que la ciencia es un tipo de actividad humana y por ello compleja y difícil de describir. Coincide con esta postura Golombek (2008), quien afirma que la ciencia es una actitud y que gramaticalmente sería más interesante considerarla un verbo y no un sustantivo: un hacer cosas, preguntas, experimentos. Cabello (2011) agrega que "aprendemos de forma activa
| La formación científica en los primeros años de escolaridad
I Panorama

I pp. 10-23

I Volumen 9

I Número 17 | Julio-diciembre | 2015 
y a estas edades es necesario, manipular, experimentar, ensayar y errar" (p. 60).

Graciela Ortiz

Martha Lucía

Cervantes |

Echeverría (1995), citado por Restrepo (2007), concibe la ciencia como actividad transformadora del mundo; un conocimiento científico, mirado desde la perspectiva de sus agentes y las personas que lo construyen, desborda el marco que le ofrece la epistemología (justificación lógica del conocimiento). Se hace necesario recurrir también a la axiología (sistema de valores que justifica las acciones humanas), puesto que la ciencia actual no solo pretende conocer el mundo sino transformarlo.

Rutherford (1990), por su parte, define la ciencia como la aplicación sistemática de valores altamente apreciados: integridad, diligencia, justicia, curiosidad, apertura hacia nuevas ideas, escepticismo e imaginación. Sostiene que la educación científica tiene una posición particularmente sólida para promover tres de estas actitudes y valores: la curiosidad, la apertura hacia nuevas ideas y el escepticismo. Afirma que los niños entran en la escuela bullendo de preguntas sobre todo lo que tienen a la vista, y solo se diferencian de los investigadores científicos porque aún no han aprendido a hallar respuestas y a verificar qué tan buenas son esas respuestas. La educación científica que promueve la curiosidad y enseña a los niños a canalizarla en forma productiva les hace un servicio tanto a los estudiantes como a la sociedad.

Porlán, García y Cañal (1997) puntualizan que el rasgo más significativo del desarrollo del currículo de ciencias durante los últimos treinta años ha sido el abandono de la enseñanza de las ciencias como un cuerpo de conocimientos establecidos (la ciencia producto o resultados), en favor de la experiencia de la ciencia como método para generar y validar tales conocimientos.

La ciencia es una forma trascendental para explorar el mundo, para develar los secretos de la naturaleza y satisfacer nuestra innata curiosidad, la ciencia es fuente para algunos de los valores éticos, aparte de las consideraciones utilitarias, la ciencia ejerce una influencia social y cultural; finalmente, la ciencia es una fascinante empresa capaz de enganchar a hombres y mujeres en lo mejor de ellos y agrandar y enriquecer el espíritu humano con sus descubrimientos (Universidad Pedagógica Nacional de México, 2000, p. 7).
Tonucci (1995) aclara que hacer ciencia no es conocer la verdad sino intentar conocerla, y sostiene la hipótesis de que los niños desde pequeños van construyendo teorías explicativas de la realidad de un modo similar al que utilizan los científicos. Por tanto, se debe propiciar en ellos una actitud de investigación que se funde en los criterios de relatividad y no sobre criterios dogmáticos. Propone el autor que hay que ayudar a los niños a darse cuenta que ellos saben que también son constructores de teorías y que son estas teorías las que deben poner en juego para saber si sirven o si es necesario modificarlas para poder dar una explicación a la realidad que los circunda.

La ciencia construida en la escuela debe ser, según Maxwell (1986), citado por Echeverría (1995), una ciencia "sabia", aquella que tiene un objetivo y un valor para la vida humana. La ciencia implica pasar de un problema científico a un problema social, de un interés individual a un interés colectivo, del pensamiento a la acción, del conocimiento enciclopédico a la comprensión. Una ciencia así es una ciencia educadora, que se dedica a problemas relevantes, por más que sean complejos y no tengan una sola solución, y esta se valora según las acciones que implique, sus posibilidades de éxito y sus consecuencias. Según Ávila et al. (2007), la ciencia está presente en la cotidianidad de los individuos, aunque en ocasiones no sea tan evidente como la tecnología. Cuéllar (2007) sostiene que es importante que el conocimiento producido en la escuela dé la capacidad al estudiante para comprender qué sucede en su entorno y le dé competencias para poder aplicar ese conocimiento escolar a las situaciones de la vida cotidiana.

A partir de estos conceptos, la ciencia es entendida no como un conjunto de conocimientos, verdades absolutas o saberes ya establecidos, sino como una constante búsqueda de respuestas a las preguntas que permanentemente los individuos se plantean sobre su propia realidad. En este proceso, se ponen en juego un conjunto de capacidades, habilidades, destrezas y actitudes que permiten explicar el mundo natural, dar respuesta a los acertijos que están presentes en la naturaleza. En este orden de ideas, se considera en esta investigación que las habilidades básicas para "hacer ciencia" son las siguientes: la pregunta, la predicción, la formulación de hipótesis, la indagación, la construcción de inferencias, la búsqueda de evidencias, la experimentación, la obtención de conclusiones y la comunicación de resultados, entre otras. 
LA CURIOSIDAD, ACTITUD POTENCIADORA DEL PENSAMIENTO CIENTÍFICO

La curiosidad es una actitud presente en los humanos que se manifiesta con mayor intensidad en los primeros años de vida. El Diccionario de la lengua española le asigna un significado un tanto despectivo y coloquial, al definir el término curiosidad como el deseo de saber alguien lo que no le concierne, o un vicio que lleva a alguien a inquirir lo que no debería importarle o el cuidado de hacer algo con primor. Mientras que la enciclopediadelasalud.com (s. f.) define la curiosidad como la tendencia a explorar, experimentar e indagar sobre algo que se considera nuevo, como un instinto natural ventajoso para la supervivencia, ya que empuja a buscar información en el medio y a interactuar con él y que facilita la creatividad. Es cualquier comportamiento inquisitivo natural que conlleve observar, examinar, indagar, averiguar, experimentar, preguntar, investigar, descubrir, inquirir, probar, escudriñar e interactuar con el mundo que hay alrededor para conseguir más conocimiento.

La curiosidad ha sido considerada como un impulso incontrolable (cuando se siente curiosidad por algo es porque se quiere conocer más sobre lo que llama la atención). Por eso se dice que la curiosidad es el inicio del conocimiento, puesto que precisamente esa necesidad de conocer es lo que ha llevado a la humanidad a desarrollar diferentes métodos de investigación para encontrar respuesta a las inquietudes.

El psicólogo investigador Berlyne (1978), considerado el padre de la curiosidad, la define como una energía que impulsa a la búsqueda del placer del conocimiento o del disfrute de los estímulos y la considera como un estado motivacional persistente que genera en los individuos comportamientos exploratorios, más notorios en unos que en otros.

Dewey (1989) definió la curiosidad como una fuerza que ayuda a desarrollar pensamiento. Considera que la educación científica se inicia desde temprana edad y comienza en la misma curiosidad y cree que esta actitud está presente en todo ser vivo como algo estremecedor ante lo nuevo. Sostiene que la función del maestro más que despertar o incrementar la curiosidad consiste en proporcionar las condiciones y los materiales a través de los cuales la curiosidad orgánica va a orientarse hasta investigaciones provistas de objetivos y hasta el incremento del conocimiento, mediante los cuales la interrogación social se transforme en la capacidad para descubrir cosas y formular preguntas cada vez más complejas.

Franco (1998) afirma que de la curiosidad natural que tienen los niños por conocer y comprender los fenómenos que los rodean nace el aprendizaje científico, y asimismo sostiene que la curiosidad es el elemento esencial de toda indagación. Afirma que los niños se muestran ansiosos por investigar, por eso la escuela debe proponer actividades que desarrollen habilidades intelectuales básicas, como la observación y la clasificación, ya que de esta manera se les da la oportunidad de interactuar con los objetos no solo para manipularlos, sino también para describirlos, compararlos y clasificarlos. Sostiene que el deseo de conocer el mundo está motivado por la curiosidad innata del niño. De ahí la importancia de proporcionarle los estímulos necesarios en cada etapa de su evolución y crecimiento.

Para las investigadoras Villate y Román (2009), la curiosidad es un proceso cognitivo que inicialmente se muestra como un impulso natural de la persona para interactuar con su entorno, cuestionarse frente a él y explorarlo. Ese impulso se convierte posteriormente en una característica permanente que es usada en forma consciente por el individuo y que está orientada hacia la obtención del conocimiento.

Tonucci (2006) sostiene la hipótesis de que los niños desde pequeños van construyendo teorías explicativas de la realidad de un modo similar al que utilizan los científicos. Sostiene que no es necesario decirles cómo es el mundo, ellos lo muestran tal como lo ven, con su propia lógica. Son los docentes quienes deben promover actitudes investigativas y exploratorias a los niños mediante su curiosidad. De esta manera, se introduce un poco de ciencia en las aulas preescolares, sin que necesariamente haya que realizar investigaciones demasiado profundas ni minuciosas. Sin embargo, las preguntas de los niños, la curiosidad, el interés, la motivación, las características del grupo, sus competencias cognitivas y la intervención docente a través de modalidades adecuadas serán algunos elementos que ayuden a trabajar la ciencia en las aulas preescolares. De este modo, tal como afirma Obando (2011), la ciencia se convierte en un pretexto para que los estudiantes asuman un papel
La formación

científica en los

primeros años de escolaridad

\section{(a)}

(n)

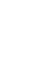


de investigadores, y experimentar, preguntar e indagar son acciones que permiten desarrollar la curiosidad científica.

Graciela Ortiz

Martha Lucía

Cervantes |

Si los científicos se aproximan al conocimiento a partir del análisis de sus observaciones, de las preguntas, conjeturas e hipótesis nacidas de su curiosidad, se puede pensar entonces que los niños también tienen la percepción de un científico investigador, porque igualmente son muy curiosos, muestran gran interés por conocer su entorno, formulan preguntas constantemente y plantean hipótesis para explicar los fenómenos que perciben.

\section{LA FORMACIÓN CIENTÍFICA EN LOS PRIMEROS AÑOS}

En los últimos años, han aumentado los escritos y las reflexiones en torno a la enseñanza de las ciencias en la escuela, sobre todo en los primeros años. Cada día se es más consciente de la importancia de tener una sólida formación científica desde la educación inicial, que despierte en los niños el interés por la ciencia y los encamine por el mundo de la investigación. Pese a esto, aún la ciencia es concebida como exclusiva de intelectuales o superdotados, vestidos con bata blanca, encerrados en laboratorios, inventando soluciones a los grandes problemas que amenazan a la humanidad.

Ávila et al. ( 2007) sostienen que en el imaginario colectivo la idea que se tiene de ciencia se fundamenta en el trabajo de científicos muy especializados que ejecutan complejos procesos a los que solo tienen acceso un selecto grupo de personas lejanas a toda cotidianidad, y a los niños se les suele presentar la ciencia como algo desvinculado de la vida cotidiana. Para Massarani (2004), los programas de televisión para niños en general representan a los científicos como personajes locos y descuidados, vestidos con bata blanca, cuyo trabajo es inventar cosas sin ninguna aplicación o diseñar aparatos o sustancias para destruir al mundo con sus inventos.

En las aulas de preescolar, se sigue concibiendo la ciencia como un área más que se transmite mediante conceptos elementales, y algunas veces abstractos, alejados de la realidad y de los verdaderos intereses de los niños sin tener en cuenta que, tal como lo afirman Porlán y De Alba (2012), no existe una única opción posible a la hora de decidir el tipo de contenidos que se van a trabajar en la escuela, así la tradición indique lo con- didáctico dominante en la enseñanza de la ciencia es el transmisionista. Dentro de este modelo, la ciencia es la acumulación de conceptos descriptivos, explicativos y comparativos de fenómenos. Así, aprenderla supone memorizar los conocimientos acumulados a lo largo de la historia, en cuya forma pedagógica se privilegia la retención de conceptos por parte de los alumnos, frente a la construcción de procesos significativos y de actitudes positivas hacia la ciencia. Por esto se deja de aprovechar la curiosidad natural que caracteriza a los niños y se desestimula su interacción con los elementos del entorno por considerar que se atenta contra un orden preestablecido en las aulas de clase.

Ruiz, Martínez y Parga (2009) afirman que, en ocasiones, la ciencia en la escuela se limita al desarrollo de algunas experiencias de laboratorio, y Massarani (2004) agrega que en muchos casos los libros didácticos son las únicas fuentes de información relacionados con la ciencia. Las acciones de los docentes en el aula tienen mucho que ver con sus creencias y, por eso, "conciben lo que es cierto en función de lo que creen que es cierto" (p. 45).

Para Segura (2013), la escuela es ante todo una oportunidad para que el niño logre experiencias de comprensión de su realidad y con ello la enriquezca, entonces lo que se estudia en el aula no será lo que ya estaba hecho y consignado en los textos, sino lo que podemos hacer articulando las actividades de aula con los problemas del entorno y de la vida. A propósito de esto, Corchuelo y Catebiel (2005) expresan:

Resulta claro que para cumplir los objetivos, en las aulas de clases de la educación se deben generar procesos en los cuales los estudiantes puedan relacionar los aspectos teóricos con la actividad investigativa y su contexto social. Es decir, se requiere de la transformación del aula de clases en un espacio que promueva la participación ciudadana en función de la construcción de alternativas de solución a problemas sociales relevantes del entorno. Entonces, se considera imprescindible construir una propuesta alternativa que supere las limitaciones de los desarrollos curriculares orientados a la transmisión de la información, y tenga en cuenta el potencial y las características particulares de los estudiantes, de los docentes y de las instituciones (p. 7). 
Claxton (1994) afirma que uno de los obstáculos más grandes para promover la conciencia del papel que tiene la ciencia en la vida escolar es considerar al niño como un sujeto limitado cognitivamente para el desarrollo de las abstracciones científicas, partiendo del déficit y la descalificación. Al analizar este panorama escolar, se confirman las aseveraciones de Porlán y De Alba (2012), cuando afirman que "la escuela es una institución adulta, pensada para los menores, pero sin los menores” (p. 7).

Tonucci (1995) plantea que, cuando se piensa que enseñar ciencia a los niños pequeños es difícil, se debe considerar que los niños desde pequeños van construyendo teorías explicativas de la realidad que viven y conocen, de un modo similar al que utilizan los científicos, a través de la experimentación, del tanteo y del error. De hecho, es el método de la ciencia; se trata de reelaborar creativamente la información que se posee, probar, intentar, verificar, comparar... entendiendo que hacer ciencia no es conocer la verdad sino intentar conocerla. El autor considera que esto lo hace el niño desde que nace, hasta que la escuela interrumpe ese modo de conocer imponiéndole otro, ajeno y clasista y determinado por opciones políticas de clases dominantes que imponen un modelo escolar literario y verbalista.

También sostiene el autor que en la formación científica durante los primeros años se cometen dos grandes errores: presentar la ciencia con conceptos demasiado simples o presentar la ciencia con conceptos muy complejos, pero de un modo simple. Muchas veces se transmiten conocimientos verdaderamente complejos sobre temas que ni siquiera el docente comprende, lo que ocasiona la enseñanza de grandes errores como si fueran verdades absolutas.

Por eso Tonucci (2006) propone que en el campo de las ciencias la escuela debe permitirle al niño el contacto con la naturaleza y con los elementos y objetos orgánicos, hoy de hecho prohibido, impedido, vedado por el acelerado e inhumano desarrollo de la ciudad. A partir de estas experiencias, el niño aprenderá a observar, escuchar, a formular las primeras hipótesis, a contrastarlas con las de los otros, a arriesgarse a las primeras teorías, a reconocer las superadas, etc. Insiste en que se debe propiciar en los niños una actitud de investigación que se funde sobre los criterios de relatividad y no sobre criterios dogmáticos. Es decir, ayudar a los niños a darse cuenta de que ellos saben, que ellos también son constructores de teorías y que es esta teoría la que deben poner en juego para saber si les sirve o si es necesario modificarla, a fin de poder dar una explicación a la realidad que los circunda.

Por su parte, Torres et al. (2013) consideran que la enseñanza de las ciencias debe apoyarse en estrategias didácticas alternativas de indagación que faciliten la participación activa del estudiante en la construcción y apropiación del conocimiento.

Se puede afirmar entonces que sí es posible que los niños en edad preescolar desarrollen un pensamiento científico. Según Shepherd (2006), citado por Tierrablanca (2009), ellos a su corta edad se formulan explicaciones acerca de lo que sucede en la naturaleza, tal vez no aceptadas por el saber científico, pero eso no les resta valor, por el contrario, son construcciones de los niños basadas en sus experiencias y en las observaciones que realizan. Cabello (2011) afirma: "En la vida cotidiana de los niños hay infinidad de vivencias que pueden favorecer una actitud científica hacia el conocimiento" (p. 58). Es decir que el pensamiento científico no es exclusivo de grandes inventores, sino que es una actitud ante el mundo que posibilita a los niños explicarse los fenómenos que observan, partiendo siempre de lo cercano, para que esas experiencias le resulten más interesantes, ya que son más próximos a su realidad.

Shepherd (2006), citado por Tierrablanca (2009), propone unos argumentos que justifican la existencia de un pensamiento científico infantil. El primero de ellos es su capacidad de preguntar, pues precisamente la pregunta es el motor del pensamiento científico, acompañada del asombro y la curiosidad. Un ejemplo de esto es el del físico Albert Einstein, quien reconocía que las preguntas que lo llevaron a hacer los descubrimientos y aportaciones que le valieron el Premio Nobel eran las mismas que se hizo de niño. En alguna ocasión, manifestó: "No tengo talentos especiales, solo soy profundamente curioso" (Laurenz, 2013, p. 26).

Otro argumento es que la mente del niño como la del adulto creativo está dispuesta a adentrarse en lo desconocido. Les encanta resolver acertijos, buscar e indagar, se asombran, se ensimisman con lo que llama su atención y además intentan explicarlo, encontrarle sentido. La curiosidad y el asombro son característicos del
| La formación

científica en los

primeros años de escolaridad 
pensamiento científico, los niños se plantean problemas que un adulto normal nunca se detiene a examinar.

Graciela Ortiz

Martha Lucía

Cervantes |

Un último argumento que rebate el autor es la idea de que la percepción de los niños pequeños es deficiente, pues a menudo la que tienen sobre un fenómeno es distinta de la que tiene la educadora. Esto sin tener en cuenta que la percepción tiene una intencionalidad que puede no ser la misma para la educadora y para los niños. Entonces se considera que el niño realmente tiene una percepción deficiente del mundo, pues no es capaz de percibir adecuadamente lo que se quiere que observe.

A partir de estos argumentos, concluye que la posibilidad de que los niños en educación preescolar desarrollen un pensamiento científico es una realidad, en cuanto los educadores piensen que tienen todas las capacidades y habilidades para hacerlo con una orientación educativa adecuada que conceptualice la niñez como un espacio de creación cognitiva, con procesos mentales que comparten paralelismos con los adultos creativos.

Glauert (1998) establece que algunas metas de la ciencia para los niños pequeños pueden ser construir y favorecer ideas e intereses en los niños, incrementar la comprensión de los niños sobre su medio ambiente físico y biológico e identificar su lugar en él, promover la conciencia del papel que tiene la ciencia en la vida cotidiana, ayudar a los niños en sus interacciones con el mundo (por ejemplo en relación con la salud y la seguridad), hacer que las cosas funcionen o cuidar a los seres vivos, estimular un pensamiento crítico, el respeto a las evidencias y el interés por el medio ambiente, desarrollar actitudes y acercamientos positivos para aprender $\mathrm{y}$ apoyar a los alumnos para que aprendan a aprender.

Glauert (1998), citado por Caravaca (2010), plantea algunas sugerencias que debe seguir el docente cuando trabaja la ciencia en el aula:

3. Demostrar interés por el medio ambiente y, sobre todo, valorar y escuchar cuidadosamente las ideas de los niños.

4. Hacer preguntas, estar preparado para someter a prueba sus ideas y cometer errores.
Finalmente, tal como lo afirmó Golombek (2008), "la única forma de aprender ciencias es haciendo ciencias" (p.10).

El aula, sea la de alumnos de escuelas primarias, sea la de los institutos de formación docente, puede y debe transformarse en un ámbito activo de generación de conocimiento, alejado de la mera repetición de fórmulas y basado en la experimentación e indagación constantes:

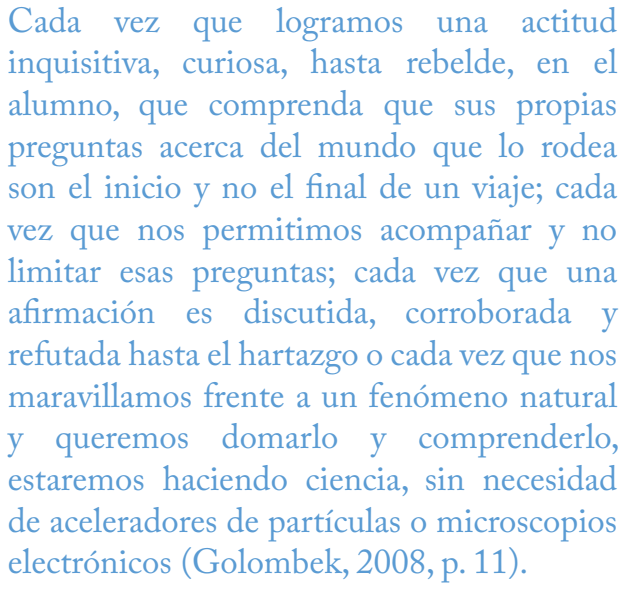

En Colombia, a pesar de que se han hecho propuestas de programas y proyectos muy serios para trabajar la ciencia e incentivar procesos investigativos en la escuela con los niños desde la educación inicial por parte del MEN con el apoyo del Departamento Administrativo de Ciencia, Tecnología e Innovación (Colciencias) y algunas instituciones de educación superior públicas y privadas y de que ha habido una gran inversión de recursos económicos y humanos, el acercamiento a la ciencia en la realidad de las aulas no se ha visto reflejado como una práctica educativa generalizada. Han faltado lineamientos claros por parte del Estado y no se ven políticas que definan, regulen, apoyen y aseguren el desarrollo general de habilidades científicas en la población infantil desde su ingreso al sistema formal de educación.

En líneas generales, no se le ha dado a la ciencia el valor y la importancia requerida y encomendada por organismos como la Unesco, mediante la aplicación de políticas, lineamientos y estándares que estimulen la investigación científica en la escuela en especial en las aulas de preescolar y sobre todo ha faltado compromiso y rigor en las instituciones educativas para desarrollar procesos y habilidades científicas en los niños mediante proyectos investigativos. Esta situación ha conllevado que se desaprovechen condiciones particulares de esta etapa 
del desarrollo del niño, como la facilidad para aprender, la búsqueda constante de información mediante preguntas, la actitud permanente de explorar e investigar y especialmente la insaciable curiosidad ante el mundo que lo rodea. Probablemente la escuela se siente comprometida en desarrollar y dedicar más tiempo a otras áreas no menos importantes, como las matemáticas y el lenguaje, que han sido promovidas y privilegiadas ancestralmente aun desde los mismos programas de formación docente. En este punto, es oportuno señalar los planeamientos de Gallego, Castro y Rey (2008), quienes sostienen que se hace necesario recorrer nuevos caminos de formación docente enfocados a las nuevas generaciones de niños y niñas para complementar y enriquecer las experiencias educativas.

Los cambios en la concepción de la ciencia en el aula implican el "fortalecimiento profesional de docentes en ejercicio y profesores en formación” (Ruiz, Parga y Martínez, 2009, p. 45). Por eso, la invitación es a las maestras y los maestros de preescolar y a quienes intervienen en la educación inicial a derrocar esos viejos paradigmas que conciben la ciencia como algo muy propio de adultos y de recintos de laboratorio, a realizar proyectos de investigación con los niños, a analizar y reflexionar sobre los resultados de las experiencias y a decidirse de una vez por todas a llevar a cabo esos cambios en las prácticas pedagógicas que tanta falta están haciendo.

\section{DesarRollo de habilidades CIENTífICAS EN LOS NIÑOS}

Las habilidades científicas son llamadas también habilidades del proceso científico, habilidades investigativas, habilidades básicas para investigar o habilidades de pensamiento científico. En general, todas se refieren a las habilidades para resolver problemas de la vida en cualquier ambiente. Sordo (2006) considera que las habilidades científicas son las cosas que hacen los científicos cuando estudian e investigan cómo observar, medir, inferir, predecir y experimentar.

Glauert (1998) afirma que la ciencia proporciona oportunidades para desarrollar habilidades asociadas a la investigación científica. Además, plantea algunas ideas que se deben tener en cuenta en la formación científica de los niños: primero, el desarrollo de las habilidades de los niños en la ciencia no puede ser dejada por completo Figura 1. Resumen de los resultados del primer y segundo ciclo. al azar. Para este autor, los niños necesitan encontrar sentido a las ideas científicas e identificarse con los procedimientos científicos por sí mismos, y los adultos tienen un papel vital en este proceso. Se puede ayudar a los niños a construir la confianza en sí mismos como aprendices, al impulsarlos a hablar acerca de sus ideas, revisar cómo realizan sus investigaciones, debatir lo que significan sus resultados y reflexionar sobre lo que han aprendido.

Segundo, es importante reconocer que los conceptos y los procesos están totalmente ligados en el aprendizaje de la ciencia. Si las actividades de las investigaciones se enfocan a desarrollar habilidades o procesos sin tomar en cuenta los conceptos teóricos que las fundamentan, pueden llegar a convertirse simplemente en actividades matemáticas, artísticas o del lenguaje.

Tercero, los niños aprenden mejor cuando las actividades se desarrollan en un contexto significativo que se relaciona con su vida cotidiana y con sus experiencias. Comenzar con las ideas de los niños y sus preguntas, usar historias familiares, entender las experiencias de los padres o de una comunidad más amplia, son algunos de los caminos para asegurar no solo que las actividades sean significativas, sino que los vínculos se construyan entre la ciencia y la vida cotidiana de los niños.

La interacción directa con el entorno, la observación y el diálogo con otros les permite a los niños la posibilidad de plantearse preguntas que dan origen a proyectos de investigación, permitiendo así que los maestros transformen sus prácticas pedagógicas (Obando, 2011). Aunque la actividad práctica es importante, también lo son la plática, el pensamiento y la imaginación. El desarrollo de habilidades científicas en los niños desde temprana edad propicia avances significativos en su aprendizaje, que los educadores pueden evidenciar. Glauert (1998) asegura que, a medida que los niños ganan experiencia, es posible que cada vez más:

1. Hagan preguntas y sugieran ideas.

2. Hagan predicciones y explicaciones basadas en conocimientos y experiencias previas.

3. Diseñen exploraciones e investigaciones más sistemáticamente, comiencen a usar las mediciones y a reconocer la necesidad de hacer pruebas confiables.
| La formación

científica en los

primeros años de escolaridad 
4. Identifiquen patrones en sus observaciones.

5. Sean capaces de comunicar los hallazgos de varias formas.

Graciela Ortiz

Martha Lucía

Cervantes |

6. Establezcan vínculos entre una situación y otra y comiencen a aplicar ideas en nuevas situaciones.

7. Muestren confianza e independencia en su acercamiento a las actividades de la ciencia.

Estos argumentos confluirían en el logro de uno de los fines de la educación establecidos en la Ley General de la Educación: "El acceso al conocimiento, la ciencia, la técnica y demás bienes valores de la cultura, el fomento de la investigación y el estímulo a la creación artística en sus diferentes manifestaciones" (Congreso de Colombia, 1994). También la Ley establece que uno de los objetivos de la educación preescolar es "el estímulo de la curiosidad para observar y explorar el medio natural, familiar y social", señalamiento que por diferentes razones se olvida en las prácticas educativas de educación inicial.

En esta perspectiva, se considera que la escuela debe orientar procesos que lleven a los niños a desarrollar desde su ingreso a la educación formal algunas habilidades científicas, como la inferencia, la clasificación, la observación, la formulación de preguntas y el planteamiento de hipótesis (MEN, 2009) que los llevará necesariamente por el camino de la investigación.

\section{LA INFERENCIA}

La Inferencia se pone en funcionamiento cuando se tiene una actividad reflexiva sobre los objetos, las situaciones y los fenómenos, de manera que los niños pueden extraer conclusiones sobre algo que no es directamente observable, a partir de información que ya se ha obtenido. La inferencia permite a los niños ir más allá de la información dada por un fenómeno que ellos desean comprender, de esta manera amplían su conocimiento, ejercitan sus habilidades de averiguación, hacen búsquedas sistemáticas, analizan la información y organizan sus ideas en la generación de soluciones y de explicaciones, en distintos contextos, incluso el educativo.

Puche (2003), citado por Orozco (2004), encontró que desde los 3 años y medio, pero sobre todo desde los 4 años, el funcionamiento inferencial parece formar parte de la actividad mental del niño al enfrentarse a los textos. capacidad para realizar inferencias aparece tempranamente. Alrededor de los 3 años los niños son capaces de realizar inferencias, pero, a medida que aumenta la edad, se da un incremento en la comprensión de la situación, lo cual hace que alrededor de los 5 años se consolide la realización de inferencias con respuestas más precisas que logran tomar todos los elementos necesarios para su ejecución.

Por otro lado, las investigaciones realizadas por Duque y Correa (2011) muestran resultados que indican que los niños de preescolar de 5 años tienen la capacidad para formular inferencias de diferente complejidad y tipología, haciéndose más fácil, como era de esperar, las de menor complejidad.

\section{LA CLASIFICACIÓN}

Esta es una habilidad de muy temprana aparición en los niños, que les permite elaborar diferentes tipos de criterios para organizar el conocimiento y la información que se tiene de los fenómenos y objetos del mundo. La clasificación consiste en organizar información y datos en grupos con características comunes y contribuye a la elaboración de categorías y conceptos (Pasek y Matos, 2007). Es gracias a este funcionamiento que una persona puede llegar a conformar un sistema coherente para establecer diferenciaciones y semejanzas entre los objetos o entre los fenómenos.

Al realizar procesos de clasificación, los niños crean sus propios criterios. Por ejemplo, al pedirles que clasifiquen tarjetas con figuras de animales, ellos pueden emplear diversos criterios de clasificación. Pueden agrupar animales por su tamaño (los grandes a un lado y los pequeños al otro), por su color (los verdes, los cafés y los amarillos), por su tipo taxonómico (los mamíferos en un grupo y las aves en otro), por el tipo de alimentos que consumen (estos comen hierbas y estos comen carne). Todas estas formas de clasificación son igualmente válidas y es el propio niño quien crea, de manera activa, las condiciones en virtud de las cuales los objetos pueden ser comparados.

\section{LA OBSERVACIÓN}

Una de las primeras habilidades que debe desarrollar un científico es la observación. La observación es un 
registro sistemático válido y confiable de comportamientos, fenómenos o hechos (Pasek y Matos, 2007). Va más allá del ver o sentir, aunque influye el empleo de sentidos para obtener información, también incluye otros aspectos que no se reducen a la simple captación. Se trata de una actividad mental y no de la mera respuesta de los órganos sensitivos a los estímulos.

Es necesario orientar a los niños en sus procesos de observación para que utilicen sus sentidos adecuadamente y con seguridad, a fin de obtener de su entorno la información necesaria para sus investigaciones, lo cual implica el desarrollo gradual hacia la selección de lo relevante de entre lo irrelevante en el contexto de una determinada investigación o problema. A los niños se les dificulta hacer este tipo de distinciones y pueden dejar de lado información importante, si reducen el espectro de sus observaciones antes de tiempo.

En los primeros años, se debe animar a los niños para que hagan cuantas observaciones puedan, prestando atención a los detalles y no solo a las características que saltan a la vista, en especial si son objetos de su interés, "haciéndoles ver los fenómenos y las situaciones experimentales de una forma especial" (Cabello, 2011, p. 59).

Es importante que los niños inicialmente hagan gran cantidad de observaciones de las semejanzas y diferencias y presten atención a los detalles tanto como a las características más llamativas (Harlen, 2001). Esto con el propósito de agrupar o clasificar. Se observa, se estructura y se categoriza lo que se percibe como parte del proceso de percepción.

Una forma de desarrollar la habilidad de observación en los niños es mediante la exploración. Esta es otra forma activa de observar, de conocer algo, más cuando se trata de un fenómeno inicial o de un fenómeno con el cual no se puede experimentar. La observación proporciona a los niños oportunidades para interactuar con objetos y materiales, les permite observar lo que ocurre y obtener una percepción mejor del fenómeno. Este proceso frecuentemente lleva a formular preguntas que pueden generar investigaciones.

\section{LA FORMULACIÓN DE PREGUNTAS} pero de lo único que no se puede hablar es que las pre-
Ante estas consideraciones cabe preguntar: ¿será que dejan de preguntar los niños porque no tienen curiosidad, o es que ya no tienen curiosidad porque dejaron de preguntar? Tonucci (2008) sostiene que en la escuela

Las investigaciones nacen de una pregunta, una duda o una incertidumbre. En este orden de ideas, se puede afirmar que la ciencia se nutre de las preguntas. El proceso de indagación implica observar detenidamente la situación, plantear preguntas, buscar relaciones causa-efecto (Narváez, 2014). Los niños están llenos de dudas y de inquietudes que son expresadas mediante un interrogante con el que esperan encontrar una respuesta. Son precisamente las respuestas que ellos mismos encuentran a través de su experiencia y sus vivencias las que generan mayor aprendizaje.

Las preguntas permiten al niño expresar sus inquietudes, curiosidades, intereses y su afán por conocer el mundo. Por eso estas deben ser escuchadas, respetadas y tenidas en cuenta desde su misma complejidad e individualidad. La búsqueda de respuesta a través de las preguntas es fundamental para el desarrollo de su pensamiento científico. Las preguntas están directamente ligadas a las competencias comunicativas. Cuando los niños formulan interrogantes o plantean hipótesis, deben hacerlo en forma clara y coherente para que estas puedan ser entendidas y respondidas.

La información hallada, ya sea mediante su propia búsqueda, ya se con ayuda de los adultos, les permite elaborar hipótesis y construir conocimiento. Pero en general la escuela enseña al estudiante lo que quiere que este aprenda y muchas veces esto no coincide con lo que el estudiante quiere aprender, precisamente porque no se han tenido en cuenta ni escuchado sus preguntas.

Tonucci (1995) sostiene que, si los niños permanentemente generan inquietudes, despiertan curiosidades. ¿Por qué cerrar la puerta entonces dándoles una respuesta? Los maestros tienen dos caminos, afirma el pedagogo, cerrar esa puerta abierta con una respuesta que los niños deben creer y aprender, o bien abrir otras puertas para que ellos puedan encontrar la respuesta o acercarse a ella. Se puede hablar de que los niños hacen preguntas ingeniosas, preguntas tediosas, preguntas mal formuladas, repetidas y muchas otras según la ocasión, guntas de los niños sean estúpidas (Sagan, 2011).

I Panorama

I pp. 10-23

I Volumen 9

I Número 17 I Julio-diciembre । 2015 
Graciela Ortiz

Martha Lucía Cervantes I

el niño aprende a callarse y se calla toda la vida. Por su parte, Trujillo (2001) considera que, cuando el niño ingresa al colegio, se comprueba progresivamente la desaparición de una actitud que consiste en querer conocer las cosas por sí mismo y, por el contrario, se estimula una actitud que invita a memorizar lo que dicen los libros o los profesores. Esto ocasiona que la capacidad espontánea, natural de investigación del niño se vea frustrada, que podría traducirse en una falta de confianza en sí mismo, llevando rápidamente a la pasividad. Los alumnos se limitan a recibir información de los profesores y aumenta el desinterés.

\section{EL PLANTEAMIENTO DE HIPÓTESIS}

Las hipótesis son suposiciones, conjeturas o predicciones lógicas que se plantean sobre lo que se espera que suceda con un objeto, un evento o un fenómeno, con el fin de dar una respuesta a un problema, contemplando múltiples posibilidades y basándose en los conocimientos previos que se tienen sobre dicho evento. "Los estudiantes pueden transitar gradualmente hacia niveles explicativos más complejos y aventurar predicciones hacia el comportamiento de los fenómenos naturales que buscan interpretar" (Acher, 2014, p. 67). Deming (1994), citado por Osorio (2009), sostiene que los niños tienen la capacidad de predecir hechos, predecir no es adivinar, y esta predicción puede basarse en una observación detallada o en una hipótesis.

Las hipótesis no son verdades inamovibles. Son afirmaciones que pueden ser aceptadas o rechazadas. E1 objetivo es dar una explicación a un problema, contemplando múltiples posibilidades, basándose fundamentalmente en los conocimientos previos que se tienen sobre dicho evento.

Las hipótesis tienen una estrecha relación con la imaginación, ya que estas son conjeturas o relaciones imaginadas sobre cómo puede ser el mundo. Si las personas no se atrevieran a plantear hipótesis sobre los fenómenos, no avanzarían en la comprensión del mundo. Los niños interiorizan sus experiencias de una forma propia para construir sus propios significados (Cabello, 2011). Lo hacen desde pequeños y progresivamente van adquiriendo la capacidad de curiosear y de relacionar eventos para predecir resultados.
El MEN (2009) define la hipótesis como el funcionamiento que permite plantear explicaciones a fenómenos o eventos que suceden en el mundo, y es la que da origen a los procedimientos científicos. Ferrater (2001), citado por Restrepo (2007), afirma que las hipótesis son enunciados que se asumen sin prueba experimental. Las suposiciones son verdaderas hipótesis, consideradas también como enunciados teóricos. Las hipótesis se diferencian de los axiomas en cuanto estos últimos se admiten como verdades evidentes, mientras que las hipótesis se asemejan más a postulados en el sentido de postulaciones. Ordoñez (2003), citado igualmente por Restrepo (2007), afirma que las hipótesis son la esencia de la racionalidad científica y que, en gran parte, el progreso de la ciencia depende de ellas.

En la escuela, el niño comienza a elaborar sus propios esquemas mentales con los que va construyendo y refinando sus conocimientos, muchos de ellos son hipótesis o suposiciones creadas por él mismo en su necesidad de comprender la realidad que lo circunda. Tonucci (1995) afirma que los niños desde pequeños van construyendo teorías explicativas de la realidad de un modo similar al que utilizan los científicos. Su constante búsqueda de respuestas y su curiosidad lo llevan permanentemente a formular hipótesis ante los diferentes fenómenos.

\section{A MODO DE CONCLUSIÓN}

Se hace necesario dar una nueva mirada a la ciencia en la escuela, dejando de concebirla como un cúmulo de conocimientos o contenidos programáticos que el estudiante debe asimilar. Es allí donde la ciencia debe asumirse como una constante búsqueda de respuestas a las preguntas que los individuos se plantean sobre la propia realidad, no solo para conocer el mundo, sino para transformarlo. Tal y como se expresa en los Estándares básicos de competencias en ciencias, "la escuela debe constituirse en un laboratorio para formar científicos", donde se reconozca la importancia que tienen las ciencias naturales en el desarrollo del conocimiento, al igual que otras áreas a las que se les asigna y dedica mayor tiempo e interés en la planeación de currículos y programas.

En las ciencias, al igual que en las demás áreas de la enseñanza, el maestro debe ser un facilitador que propone y no que impone. Debe guiar al niño para que avance, brindándole la oportunidad de equivocarse o de 
saber que está equivocado y de encontrar él mismo la verdad.

Además el trabajo de la ciencia en el preescolar no solo permite trabajar en los niños el lenguaje oral y gráfico, sino que desarrolla en ellos competencias comportamentales, como trabajo en equipo, normas de comportamiento y educación ambiental, entre otras.

En este momento en el que se vive una cultura competitiva y de cambio permanente, donde el desarrollo de los pueblos se determina por sus progresos tecnológicos y sus avances en ciencia e investigación, son el Estado y la escuela los que deben asumir el compromiso de formar ciudadanos capaces de responder a estos retos. Este proceso contribuirá a que el país se construya potenciando habilidades y actitudes científicas en los ciudadanos que, además, les permitan valorar la ciencia como una parte fundamental para el desarrollo humano y la calidad de vida (Hillerns, Morales y Cerda, 2005). Esto es un verdadero desafío que coloca en manos de los maestros la responsabilidad de fomentar y orientar la formación científica en los niños desde su ingreso al proceso de educación formal. Solo así se podrá pensar que el mejoramiento de la calidad en la educación es una realidad que puede y debe generarse en las aulas de clases.

\section{REFERENCIAS BIBLIOGRÁFICAS}

1. Acher, A. (2014). Cómo facilitar la modelización científica en el aula. Tecné, Episteme y Didaxis: Revista de la Facultad de Ciencia y Tecnologia, 36, 63-75.

2. Ávila, F., Bautista, S., Betancour, J., Calderón, J., García, C. y Triana, R. (2007). Ciencia y cotidianidad, competencias culturales básicas. En X Reunión de la Red de Popularización de la Ciencia y la Tecnología en América Latina y el Caribe (RED POP-Unesco) y IV Taller: Ciencia, comunicación y sociedad. San José, Costa Rica.

3. Berlyne, D. (1978). Curiosity and Learning. Motivation and Emotion, 2(2), 97-175.

4. Cabello Salguero, M. ${ }^{\mathrm{a}}$ J. (2011). Ciencia en educación infantil: la importancia de un "rincón de observación y experimentación" o "de los experimentos" en nuestras aulas. Pedagogía Magna, 10, 58-63.

5. Caravaca, I. (2010). Conocimiento del entorno: acercamiento infantil al saber científico. Innovación y Experiencias Educativas, 36, 1-16.
6. Claxton, G. (1994). Ciencia para todos. En Educar mentes curiosas (pp. 11-15). Madrid: Visor.

7. Coleto, C. (2009). Filosofía de la ciencia y educación científica. Innovación y Experiencias Educativas, 45, 1-9.

8. Congreso de Colombia (1994). Ley 115. Recuperado de http://www.col.ops-oms.org/ juventudes/Situacion/LEGISLACION/ EDUCACION/EL11594.HTM

9. Corchuelo, M. y Catebiel, V. (2005). Orientaciones curriculares en el enfoque CTS para educación media. Tecné, Episteme y Didaxis, 18, 121-131.

10. Cuéllar, Z. (2007). Conocimiento escolar construido a partir del interés del estudiante para comprender el mundo. Nodos y Nudos, 3, 111-117.

11. Dewey, J. (1989). Cómo pensamos: nueva exposición de la relación entre pensamiento y proceso educativo. Barcelona: Paidós.

12. Duque Aristizábal, C. P. y Correa Restrepo, M. (2011). Inferencias sobre un texto narrativo en contextos de interacción en la educación inicial. Universitas Psychologica, 11(2), 559-570.

13. Echeverría, J. (1995). Filosofía de la ciencia. Madrid: Akal.

14. enciclopediadelasalud.com (s. f.). Curiosidad. Recuperado de http://www.enciclopediasalud. $\mathrm{com} /$ definiciones/curiosidad

15. Franco, O. (1998). Del asombro y la curiosidad a la comprensión del mundo: ¿cómo lograrlo? La Habana: CubaEduca.

16. Gallego A., Castro, J.y Rey, J. (2008). El pensamiento científico en los niños y las niñas: algunas consideraciones e implicaciones. Investigación e Innovación en Enseñanza de las Ciencias IIEC, 2(3), 22-29.

17. García, M. y Peña, P. (2002). Los encuentros científicos en preescolar. Educere, 6(19), 308-315.

18. Gil Chaves, L. (2010). Desarrollo de habilidades de pensamiento inferencial y comprensión de lectura en niños de 3 a 6 años (Tesis de grado, Universidad Nacional de Colombia).

I Panorama

I pp. 10-23

19. Glauert, E. (1998). Sciencie in the Early Years. En I. A. Siraj-Blatchford, Curriculum Development Handbook for Early Childhood Educators (pp. 77-91). Londres: Trentham Books Limited. 
Graciela Ortiz

Martha Lucía

Cervantes!

Panorama

pp. 10-23 I

Volumen 9 |

Número 17!

Julio-diciembre |

2015 I

31. Pasek, E. y Matos, Y. (2007). Habilidades cognitivas básicas de investigación presentes en el desarrollo de los proyectos de aula. Educere, 37, 349-356.

21. Harlen, W. (2001). Observación y comunicación. En La enseñanza de las ciencias naturales en la escuela primaria (pp. 117-121). México.

22. Hillerns, I., Morales, H. y Cerda, G. (2005). Influencia del aprendizaje de las ciencias en niños y niñas de educación parvularia y NB1: estudio de casos. REXE: Revista de Estudios y Experiencias en Educación, 4(7), 123-140.

23. Laurenz, M. (2013). Influencia espiritual.USA: Zondervan.

24. Massarani, L. (2004). La divulgación científica para niños. Ciencia hoje das criancas de la Sociedad Brasileña para el progreso de la ciencia, 141, 78-82.

25. Ministerio de Educación Nacional (2004). Estándares básicos de competencias en ciencias naturales y ciencias sociales. Bogotá. Recuperado de http://www.mineducacion.gov.co/cvn/1665/ articles-86313_archivo_pdf.pdf

26. Ministerio de Educación Nacional (2009). Instrumento diagnóstico de competencias básicas en transición. Bogotá. Recuperado de http://cms.univalle.edu.co/cognitiva/wp-content/archivos/publicaciones/Elementos $\% 20$ conceptuales_aprender\%20y\%20jugar.pdf

27. Narváez, I. (2014). La indagación como estrategia en el desarrollo de competencias cientificas, mediante la aplicación de una secuencia didáctica en el área de ciencias naturales en el grado tercero de básica primaria (Tesis de maestría, Universidad Nacional de Colombia, Palmira).

28. Obando, L. (2011). Tres niños preguntan: ¿cómo ha evolucionado la vida en nuestro planeta? Escritos sobre la Biología y su Enseñanza, 6(4), 182-190.

29. Orozco, B. (2004). Inferencia, comprensión de textos narrativos y formación de herramientas cientificas en niños invidentes. Cali: Universidad del Valle.

30. Osorio, A. (2009). Habilidades cientificas de los niños y las niñas participantes en el Programa de Pequeños Cientificos de Manizales: pruebas de papel y lápiz (Tesis de maestría Universidad de Manizales, Colombia).
20. Golombek, D. (2008). Aprender y enseñar ciencias: del laboratorio al aula y viceversa. En IV Foro Latinoamericano de Educación: Aprender y enseñar ciencias. Desafíos, estrategias y oportunidades.

32. Porlán, R. y De Alba, N. (2012). La escuela que queremos. Investigación en la Escuela, 77, 5-12.

33. Porlán, R., García, J.y Cañal, P. (1997). Constructivismo y enseñanza de las ciencias. Sevilla: Díada.
34. Restrepo, F. (2007). Habilidades investigativas en niños de 5 a 7 años de instituciones oficiales $y$ privadas de la ciudad de Manizales. Manizales: Universidad de Manizales.

35. Ruiz, D., Parga, D. y Martínez, L. (2009). Creencias de los profesores de preescolar y primaria sobre ciencia, tecnología y sociedad, en el contexto de una institución rural. Tecné, Episteme y Didaxis, 25, 41-61.

36. Rutherford, F. J. (1990). Science for all Americans. Oxford: Oxford University Press.

37. Sagan, C. (2011). Demon-Haunted World: Science as a Candle in the Dark. Ballantine Books.

38. Segura, D. (2013). El pensamiento científico y la formación temprana: una aproximación a las prácticas escolares en los primeros años vistas desde la ciencia y la tecnología. Infancias Imágenes, 12(1), 131-140.

39. Sordo M., V. (2006). La importancia del desarrollo de las habilidades del proceso científico en el aprendizaje de la ciencia y su aplicación en proyectos de ciencia experimental para niños. En Memoria de la Segunda Reunión Nacional de Análisis: La actividad experimental en el aprendizaje de las ciencias naturales y exactas. Culiacán, México.

40. Tierrablanca Díaz, C. (2009). Desarrollo del pensamiento científico en niños pequeños. Magisterio, 20-24.

41. Tonucci, F. (1995). El niño y la ciencia. En Con ojos de maestro (pp. 85-107). Buenos Aires: Troquel.

42. Tonucci, F. (1998). A los tres años se investiga. Hogar del Libro.

43. Tonucci, F. (2006). Desarrollo, aprendizaje y evaluación en la escuela infantil. En El proceso de evaluación en preescolar: significado implicaciones (pp.18-23). México: Guía del Taller General de Actualización.

44. Tonnuci, F. (2008). La misión principal de la escuela ya no es enseñar cosas. La Nación. Recuperado de http://www.lanacion.com. ar/1085047-la-mision-principal-de-la-escuelaya-no-es-ensenar-cosas 
45. Tonucci, F. (2009). ¿Se puede enseñar la participación? ¿Se puede enseñar la democracia? Investigación en la Escuela, 68, 18-20.

46. Torres, A., Mora, E., Garzón, F. y Ceballos, N.

(2013). Desarrollo de competencias científicas a través de la aplicación de estrategias didácticas alternativas: un enfoque a través de la enseñanza de las ciencias naturales. Tendencias, 15(1), 187-215.

47. Trujillo, E. (2001). Desarrollo de la actitud científica en niños de edad preescolar. Anales de la Universidad Metropolitana, 1(2), 187-195.

48. Unesco (1999). Declaración sobre la ciencia y la utilización del conocimiento científico. Recuperado de http://www.unav.es/cdb/ uneciencia.html

49. Universidad Pedagógica Nacional de México (2000). El niño y la ciencia. México.

50. Villate, Y.y Román,J. (2009). Caracterización de la curiosidad en niños de 10 a 12 años participantes del programa Centro Amar Kennedy a través del estudio de caso (Tesis de maestría, Pontificia Universidad Javeriana, Colombia). 